\title{
Acyl-CoA thioesterase 1 prevents cardiomyocytes from Doxorubicin-induced ferroptosis via shaping the lipid composition
}

\author{
Yunchang Liu ${ }^{1,2}$, Liping Zeng ${ }^{3}$, Yong Yang ${ }^{1,2}$, Chen Chen $\mathbb{1}^{1,2}$, Daowen Wang ${ }^{1,2}$ and Hong Wang ${ }^{1,2}$
}

\begin{abstract}
In this study, we first established the doxorubicin-induced cardiotoxicity (DIC) model with C57BL/6 mice and confirmed cardiac dysfunction with transthoracic echocardiography examination. RNA-sequencing was then performed to explore the potential mechanisms and transcriptional changes in the process. The metabolic pathway, biosynthesis of polyunsaturated fatty acid was significantly altered in DOX-treated murine heart, and Acot1 was one of the leading-edge core genes. We then investigated the role of Acot1 to ferroptosis that was reported recently to be related to DIC. The induction of ferroptosis in the DOX-treated heart was confirmed by transmission electron microscopy, and the inhibition of ferroptosis using Fer-1 effectively prevented the cardiac injury as well as the ultrastructure changes of cardiomyocyte mitochondrial. Both in vitro and in vivo experiments proved the downregulation of Acot1 in DIC, which can be partially prevented with Fer-1 treatment. Overexpression of Acot1 in cell lines showed noteworthy protection to ferroptosis, while the knock-down of Acot1 sensitized cardiomyocytes to ferroptosis by DIC. Finally, the heart tissue of aMHC-Acot1 transgenic mice presented altered free fatty acid composition, indicating that the benefit of Acot1 in the inhibition of ferroptosis lies biochemically and relates to its enzymatic function in lipid metabolism in DIC. The current study highlights the importance of ferroptosis in DIC and points out the potential protective role of Acot1 in the process. The beneficial role of Acot1 may be related to its biochemical function by shaping the lipid composition. In all, Acot1 may become a potential treating target in preventing DIC by anti-ferroptosis.
\end{abstract}

\section{Introduction}

Doxorubicin (DOX), has been reported to induce various forms of regulated cell death, including apoptosis, necroptosis, and pyroptosis, of terminally differentiated cardiomyocytes, which is a vital factor in the occurrence of myocardial injury ${ }^{1-3}$. Dox-induced cardiotoxicity (DIC) has been ascribed to disrupted iron metabolism (iron

\footnotetext{
Correspondence: Hong Wang (hong_wang1988@126.com)

'Division of Cardiology, Department of Internal Medicine, Tongji Hospital,

Tongji Medical College, Huazhong University of Science and Technology, 430030 Wuhan, China

${ }^{2}$ Hubei Key Laboratory of Genetics and Molecular Mechanisms of Cardiological Disorders, 430030 Wuhan, China

Full list of author information is available at the end of the article

These authors contributed equally: Yunchang Liu, Liping Zeng

Edited by G. Blandino
}

overload) and excesses of reactive oxygen species (ROS). Generation of ROS by iron dysregulation, which may damage DNA, protein, lipid composition of membrane structures, and eventually accelerates the death of cardiomyocytes. However, studies have witnessed the failures of most kinds of 'antioxidants' as treatments for DIC ${ }^{4,5}$. Emerging studies showed that it was not ROS scavenging but the induction of an unrevealed mitochondrial redox signaling, that casts protective effect in the mito-Q (mitochondria-targeted form of CoQ10) treatment of $\mathrm{DIC}^{6}$. In all, patterns of cell death that have not been fully elucidated may also contribute to DIC.

Ferroptosis, a novel form of regulated cell death, is characterized by an iron-dependent increase in lipid peroxidation (with lipid hydroperoxides and aldehydes 
formation) and damage of biological membrane structures $^{7,8}$. Studies have shown that the oxidation of $\omega-6$ polyunsaturated fatty acid (PUFA) based phosphatidylethanolamines (PEs) is the crucial procedure of the induction of ferroptosis and genes that influence the formation of PUFA-CoA (acyl-CoA synthetase long-chain family member 4, Acsl4) or the insertion of PUFA-CoA to PEs in cells (lysophosphatidylcholine acyltransferase 3, Lpcat3) may influence the induction of ferroptosis 9 Though most of the researches focuses on the role of ferroptosis in cancer diseases, a recent one has linked cell death in DIC to ferroptosis. It was considered that the upregulation of $\mathrm{Nrf} 2 / \mathrm{Hmox} 1$ in DIC disarranged the homeostasis of iron metabolism and increased the process of ferroptosis in cardiomyocytes ${ }^{10}$. However, few studies have investigated the role of other enzymes of PUFA metabolism-related pathways in the induction of ferroptosis.

Acyl-CoA thioesterase 1 (Acot1), as an important enzyme in fatty acid metabolism, catalyzes the reaction of fatty acyl-CoAs to CoA-SH and free fatty acids ${ }^{11}$. Due to the high energy demand to supply circulating function, cardiomyocytes prefer fatty acids as metabolism substrates and possess massive of mitochondria for oxidation $^{12}$. Mitochondria are considered to be one of the majority sources of lipid peroxidation, which prompt cardiomyocytes to gain a higher capacity for lipid-ROS production ${ }^{13}$. Our researches before have shown that Acot1 can reduce the oxidative stress in cardiomyocytes and protect cardiac function in many disease models, including LPS-induced cardiac injury and diabetic cardiomyopathy $^{14,15}$. Enzymatically, Acot1 catalyze the opposite biochemical process of which is mediated by ACSL4, thus, may perform potential anti-ferroptosis ability. Whether Acot1 contributes to the inhibition of lipid-ROS formation and ferroptosis in doxorubicin cardiotoxicity has not been investigated.

In the current study, we initially performed both in vivo and in vitro cardiac cytotoxicity models of DOX to explore the role of ferroptosis and the possible mechanism in this process. We identified that biosynthesis of polyunsaturated fatty acid was one of the most downregulated bio-pathways and Acot1 was one of the most down-regulated genes in the DOX-treated murine heart with RNA-seq based bioinformatic analysis. We hypothesized that Acot1 might play a role in DIC by protecting ferroptosis. GC-MS was then used to explore the function of modified Acot1 expression in cardiomyocytes and the molecular mechanism of Acot1 in inhibiting ferroptosis. We found that modified expression of Acot1 caused the reshape of lipid composition in murine heart and subsequently affected the sensitivity of cardiomyocytes to ferroptosis. Evidence in HL-1 cardiomyocytes cell line supported that Acot1 inhibited lipid peroxidation and prevented cells from ferroptosis in DIC. Our results provided insights into the novel pathogenic mechanism of ferroptosis in the process of DIC and possible therapeutic target for the clinical practice.

\section{Materials and methods \\ Chemicals and reagents}

Chemicals and reagents were from MedChemExpress including: Doxorubicin (\#HY-15142A, DOX), Ferrostatin1 (\#HY-100579, Fer-1), (1S,3R)-RSL3 (\#HY-100218A, RSL-3), Stearic acid (\#HY-B2219), and Arachidonic acid (\#HY-109590), Docosahexaenoic Acid (\#HY-B2167). Acot1 siRNAs and negative control were from RiboBio (China). DNA ladders, pre-stained protein markers were from Thermo Fisher Scientific (USA).

\section{C57BL/6 mice}

All animal experimental protocols were approved by the Institutional Animal Research Committee of Tongji Medical College and following the ARRIVE Guidelines. C57BL/6 male mice (20-25 g) at 7 weeks were purchased from the Vital River Laboratory Animal Technology Co., Ltd. Mice were housed under specific pathogen-free conditions. After adaptively feeding for 1 week, all the mice were divided into different groups randomly and the experiment was then conducted. No blinding was done in animal experiments.

\section{Doxorubicin-induced sub-acute cardiac injury model}

A modified protocol was used to establish an in vivo sub-acute DIC model with a $25-\mathrm{mg} / \mathrm{kg}$ cumulative dose. Briefly, two doses of DOX was administrated by intraperitoneal injection, $15 \mathrm{mg} / \mathrm{kg}$ at Day 1 , and $10 \mathrm{mg} / \mathrm{kg}$ at Day 8. Mice were then killed at Day 15 after transthoracic echocardiography examination. Murine organs were then collected, frozen in liquid nitrogen followed by storage at $-80^{\circ} \mathrm{C}$ for further experiments or fixed with $4 \%$ paraformaldehyde for the histological analysis.

\section{Echocardiography}

After anesthesia at Day 15, the myocardial function of different groups was assessed using Vevo770® highresolution imaging system with a $30-\mathrm{MHz}$ high-frequency scan-head (Visual Sonics, Canada) as previously descri$\operatorname{bed}^{16}$. And data were then analyzed with its supporting software packages.

\section{RNA-Seq analysis}

RNA samples of murine heart tissue were collected from C57BL/6 mice treated with or without DOX and stored with RNAStore solution at $4{ }^{\circ} \mathrm{C}$ (\#DP408-02, Tiangen Biotech Co., Ltd.). RNA isolation, quality control, library construction, and sequencing were performed by the Beijing Genomics Institute (www.genomics.org.cn, 
BGI) using the BGISEQ-500 platform. Cleaned reads were then mapped to the GRCm38.P6 reference genome with Bowtie2 (v2.2.5) ${ }^{17}$. Transcript abundances were measured with RSEM (v1.2.8) ${ }^{18}$. Further bioinformatics analyses were all accomplished in RStudio with $\mathrm{R}$ (v3.5.3) and GSEA (v4.0.3) with GSKB (v1.14.0) ${ }^{19-21}$. Low count genes were removed by a cut-off at 0.5829 FPKM. Differential expression analyses were performed with limma ( $R$ packages v3.38.3) by a cut-off at 1.44 -fold-change and $P$ value $<0.05^{22}$. KEGG pathway enrichment was performed with clusterProfiler $(\mathrm{v} 3.10 .1)^{23}$. Plots performed with ggplot2 (v3.2.1) and ggpubr (v0.2.4) ${ }^{24,25}$. Heatmap of 50 up-regulated genes and 50 down-regulated genes was plotted with pheatmap (v1.0.12) ${ }^{26}$. All codes are available from the corresponding author upon request.

\section{Histology staining}

Murine heart tissue samples were firstly fixed with $4 \%$ paraformaldehyde and embedded into paraffin. Tissue sections were then subjected to $4 \mathrm{~mm}$ thick for next Hematoxylin-Eosin (HE) and Sirius Red staining. Cross area of cardiomyocytes and collagen area percentages were calculated using Fiji-ImageJ (v1.52p $)^{27}$.

\section{Transmission electron microscopy}

Murine heart tissue samples were fixed with a $2.5 \%$ glutaraldehyde solution for $6 \mathrm{~h}$ at $4{ }^{\circ} \mathrm{C}$. Samples were washed three times in cold $0.1 \mathrm{M}$ phosphate buffer $(\mathrm{PH}$ 7.4) for $15 \mathrm{~min}$, then fixed for $2 \mathrm{~h}$ with $0.1 \mathrm{M}$ phosphate acid buffer (PH 7.4) containing $1 \%$ osmic acid at room temperature, and again washed three times in cold $0.1 \mathrm{M}$ phosphate buffer (PH 7.4) for $15 \mathrm{~min}$. Then samples were dehydrated with a gradient ethanol solution, infiltrated with acetone-epoxy resin, and finally embedded in epoxy resin. The samples were polymerized in a $60^{\circ} \mathrm{C}$ oven for $48 \mathrm{~h}$. Embedded samples were cut into ultrathin sections (100 nm thickness) using a Leica EM UC7 microtome. The specimens then underwent uranium-lead (3\% uranyl acetate for $15 \mathrm{~min}$ and lead lemon solution for $10 \mathrm{~min}$ ) double-staining at room temperature and dried overnight. Slides were examined with a Tecnai $\mathrm{G}^{2} 20$ TWIN transmission electron microscope (FEI, USA) at an accelerating voltage of $200 \mathrm{kV}$. Digital images were obtained using its supporting software packages.

\section{MDA concentration}

The concentration of malondialdehyde (MDA) in murine heart tissues was detected using the corresponding kits (\#A003-1-2, Nanjing Jiancheng Bioengineering Institute, China), according to the instructions. MDA concentration was normalized to protein concentrations, which were detected by the BCA method with the kit (\#AR0197, Boster Biological \&Technology Co., Ltd., China).

\section{HL-1 cell culture, transfection, and treatment}

HL-1 cells were cultured in supplemented Claycomb Medium (\#51800C, Sigma-Aldrich, USA). Before most experiments, $70-80 \%$ confluence was reached. In Acot1 knock-down experiments, HL-1 cells were plated into 12well plate and transfected with siRNAs against mice Acot1 (si-Acot1, $100 \mathrm{nM}$ ) or negative control (si-NC, $100 \mathrm{nM}$ ) independently using Lipofectamine 2000 (\#11668030, Thermo Fisher Scientific) in Opti-MEM (\#31985088, Thermo Fisher Scientific) according to the manufacturer's protocol. In Acot1 overexpression experiments, HL-1 cells were plated into 12-well plate and then transfected with pcDNA 3.1(+)-Acot1 plasmids (Acot1-OE, $200 \mathrm{ng} /$ well) or vector of pcDNA 3.1(+) (vector, $200 \mathrm{ng} /$ well) independently using Lipofectamine 2000 in Opti-MEM, according to the manufacturer's protocol. In fatty acids treatment experiments, different concentrations of Stearic acid, Arachidonic acid, and Docosahexaenoic Acid were incubated with HL-1 cells overnight. The details about the treatment of HL-1 cardiomyocytes were described. In most experiments, HL-1 cardiomyocytes were treated with $2 \mu \mathrm{M}$ DOX or $5 \mu \mathrm{M}$ RSL-3 dissolved in DMSO for $24 \mathrm{~h}$ or $6 \mathrm{~h}$.

\section{Cell viability assay}

HL-1 cardiomyocytes were seeded in 96-well plates and assays of cell viability were detected after $24 \mathrm{~h}$ of treatment by the cell counting kit-8 (CCK-8; \#P5090, Wuhan Promoter Biological Co., Ltd., China). After indicated concentrations of DOX treatment, a total of $100 \mu \mathrm{l}$ serumfree Claycomb Medium containing $10 \mu \mathrm{l}$ CCK-8 solution was added to each well and incubated at $37^{\circ} \mathrm{C}$ for $2 \mathrm{~h}$. Then the absorbance at $450 \mathrm{~nm}$ was measured with a microplate reader (BioTek Instruments, USA). Cell viability were all normalized to relative control.

\section{Intracellular glutathione measurement}

The concentration of GS-SG and total glutathione in different samples were measured using the GSH and GSSG Assay Kit (\#S0053, Beyotime Biotechnology, China) according to the manufacturer's protocol.

\section{Lipid peroxidation assessment using C11-BODIPY 581/591}

Flow cytometric: HL-1 cardiomyocytes were seeded in 12-well plates. After DOX treated for $6 \mathrm{~h}$, cells were trypsinized, washed with sterile HBSS for 2 times, and then incubated with $2 \mu \mathrm{M}$ C11-BODIPY (581/591) in serum-free Claycomb Medium at $37^{\circ} \mathrm{C}$ for $30 \mathrm{~min}$. Cells were then pelleted, washed with sterile HBSS for 2 times, resuspended in HBSS, and detected with the flow cytometer (BD Accuri ${ }^{\mathrm{TM}}$ C6, Becton Dickinson, USA). A minimum of 10,000 live cells gated with blank control was analyzed per condition. FL-1 channel was considered to 
present ox-BODIPY signal and data were then analyzed by FlowJo (v10.0.7).

Fluorescent image: HL-1 cardiomyocytes were seeded in Confocal Dishes (Coverglass Bottom Dish, Solarbio life science, China). After DOX treated for $6 \mathrm{~h}$, cells were washed with sterile HBSS two times, and then incubated with $2 \mu \mathrm{M}$ C11-BODIPY (581/591) in serum-free Claycomb Medium at $37^{\circ} \mathrm{C}$ for $30 \mathrm{~min}$. Dishes were then washed and fluorescent images were acquired with the confocal microscope (LSM780, ZEISS, USA).

\section{Western blotting}

The protocol of western blotting analysis was as previously described ${ }^{14}$. After treated HL-1 cells were harvested and protein was extracted, protein concentrations from different samples were determined with the BCA protein assay kit. Samples were fractionated by $10 \%$ SDSPAGE and transferred to activated Polyvinylidene difluoride (PVDF) membranes (Bio-Rad Laboratories, USA). Membranes were blocked with 5\% FBS in TBST (Tris-buffered saline solution with $0.1 \%$ Tween-20) for $2 \mathrm{~h}$ at room temperature and then incubated with 1:1000 TBST diluted primary Anti-ACOT1 antibody (\#ab100915, Abcam, UK). Images of visualized blot bands were acquired and Gapdh was used as the loading control.

\section{RealTime-qPCR}

The protocol of RealTime-qPCR was as previously described $^{14}$. After total RNA of treated HL-1 cells or cardiac tissues was extracted using RNAiso plus reagent (\#9108, TaKaRa, China) following manufacturer's instructions, cDNA was acquired with PrimeScript ${ }^{\mathrm{TM}} \mathrm{RT}$ reagent Kit with gDNA Eraser (\#RR047A, Takara, China). Later, qPCR was performed using an SYBR Premix Ex Taq II (\#RR820A, TaKaRa, China) to detected the targeted mRNAs levels with the 7900HT Fast RealTime PCR system (Applied Biosystems, USA) and Gapdh was used as the internal control. Each reaction was performed in triplicate, and fold change analysis was performed by the $2^{-\Delta \Delta \mathrm{Ct}}$ method.

The primer sequences involved in RealTime-qPCR are as follows:

\begin{tabular}{lll} 
Gene & Forward $\left(5^{\prime} \rightarrow 3^{\prime}\right)$ & Reverse $\left(5^{\prime} \rightarrow 3^{\prime}\right)$ \\
Gapdh & CATCACTGCCACCCAGAAGACTG & AtgCCAGTGAGCTTCCCGTTCAG \\
Acot1 & TGATGGTTtGGAGGTTGGGG & TGAAACTCCATTCCCAGCCC \\
Ptgs2 & TGGGCCATGGAGTGGACTTA & TCTCAGGGATGTGAGGAGGG \\
\hline
\end{tabular}

aMHC-Acot 1 transgenic mice

The generation of $\alpha \mathrm{MHC}$-Acot1 transgenic mice on the C57BL/6 background was described previously ${ }^{14}$. Mice 8 weeks of age were killed and heart tissues were then collected, frozen in liquid nitrogen followed by storage at $-80{ }^{\circ} \mathrm{C}$ for $\mathrm{GC}-\mathrm{MS}$ detection.

\section{Total fatty acids extraction}

The samples of the murine hearts were mixed with $2 \mathrm{ml}$ of acidified methanol and incubated at $80^{\circ} \mathrm{C}$ for $30 \mathrm{~min}$. After methyl esterification, the samples were extracted with $1 \mathrm{ml}$ of hexane and washed with $5 \mathrm{ml}$ of $\mathrm{ddH}_{2} \mathrm{O}$. Sodium sulfate was used to remove remained water. Next, $500 \mu \mathrm{l}$ supernatant of the extract was mixed with $25 \mu \mathrm{l}$ of internal standard (NU-CHEK-PREP methyl esterified fatty acids mixture added with methyl Salicylate) before injection.

\section{Free fatty acids quantification with GC-MS}

Each $1 \mu \mathrm{l}$ of the extracted sample was analyzed by GCMS operating in a single ion monitoring mode by Shanghai Applied Protein Technology Co., Ltd. In detail, separation and detection were made by Agilent 7890A/ 5975C system (Agilent) with Agilent DB-WAX column (Agilent, $0.25 \mu \mathrm{m}, 0.25 \mathrm{~mm} \times 30 \mathrm{~m}$ ). The column temperature program was as follows: (1) $50{ }^{\circ} \mathrm{C}$ for $3 \mathrm{~min}$; (2) temperature was gradually elevated to $220^{\circ} \mathrm{C}$ in $17 \mathrm{~min}$; and (3) temperature was gradually elevated to $250{ }^{\circ} \mathrm{C}$ in $2 \mathrm{~min}$ and maintained for $10 \mathrm{~min}$. Helium was used as the carrier gas, and the flow rate was set to $1.0 \mathrm{~mL} / \mathrm{min}$. Electron impact ion source (EI) was applied for Mass spectrometry assay. The temperature was set to $280{ }^{\circ} \mathrm{C}$ for injection port, $230{ }^{\circ} \mathrm{C}$ for ion source, and $250{ }^{\circ} \mathrm{C}$ for the Inlet line. QC samples (pooled sample from an equal aliquot of each sample in the experiment) were injected with SIM mode at the beginning of the MS study and after every five injections throughout the experiment, which was used to monitor the MS performance. Finally, the mass data were analyzed by MSD ChemStation to determine the concentration of each compound.

\section{Statistics}

No statistical methods were used to predetermine the sample sizes. No data exclusions were made in experimental sections and the exclusion criteria in bioinformatics analysis were shown in the corresponding sections. No data show significant deviation from normal distribution and data from different treatment groups show good homogeneity of variances. The exact sample size for each experimental group has been shown in every figure as the number of dots. Lines in each figure represent the mean value of each group. Two-tailed unpaired Student's $t$ test was used to compare the means between two different treatment groups and one-way ANOVA test with post-hoc tests (Tamhane's T2 multiple comparison test) were used to compare the means among the three or more different treatment groups. All statistical analyses were accomplished with SPSS Statistics (v24.0.0, IBM, 
USA) and GraphPad Prism (v8.0.1, GraphPad Software, Inc., CA).

\section{Results \\ DOX causes significant death and cardiac dysfunction in mice}

We first established the DOX-induced sub-acute cardiac injury model using C57/BL6 mice with DOX intraperitoneal injection and measured mice survival for 14 days (Fig. 1a, b). The body and heart weight were measured after killing. As assumed, DOX treatment caused more mice death comparing with vehicle control. DOX-treated mice show lighter body and heart weight, as well as lower Heart/Tibia index comparing with control mice (Fig. 1c). Before euthanasia, the cardiac function of mice was measured with Transthoracic Echocardiography. DOX-treated survived mice demonstrated significant reduction of cardiac systolic function, as suggested by significantly reduced Ejection Fraction (EF), Fractional Shortening (FS), and increased systolic Left Ventricular Internal Diameter (LVIDs) in comparison with control (Fig. 1d, e). These data reveal that DOX causes cardiac injury in mice, mainly affects the systolic function of left ventricular and increases mortality.
RNA-seq analysis reveals the downregulation of Acot1 dominated biosynthesis of unsaturated fatty acid in DOXtreated murine heart

RNA-sequencing (RNA-seq) analysis was performed to determine the differentially expressed genes using DOXtreated and control murine hearts, to further explore the potential molecular mechanisms. MA-plot and Volcano-plot were performed to identify the significantly regulated genes with a middle-high expression basement (Fig. 2a, b). The genes in the intersection were then used for enrichment analysis. (Fig. 2c). The most regulated genes, with 50 upregulated and 50 down-regulated, show good consistency in each sample (Fig. 2d). Expanded lists of differentially expressed genes were provided (Supplementary Tables 1 and 2). In KEGG-pathway enrichment analysis, the biosynthesis of unsaturated fatty acids was one of the most significantly down-regulated metabolic pathways, which containing Acot1, Acot2, Acot3, Scd2, and Scd4 (Fig. 2e). GSEA analysis also confirmed the same pathway negatively related enriched after DOX treatment and Acot1 as one of the leading-edge core genes in the gene set (Fig. 2f). Protein-Protein Interaction (PPI) network analysis was performed with the significantly changed genes (Fig. 2g). Real-time PCR showed that DOX treatment led to a significant decrease in mRNA levels of listed

A

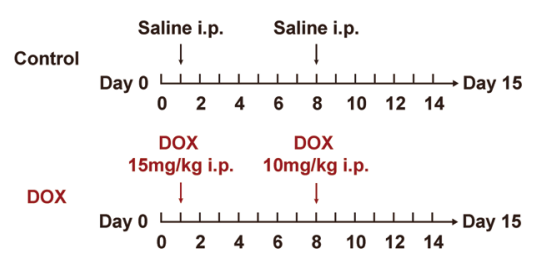

B

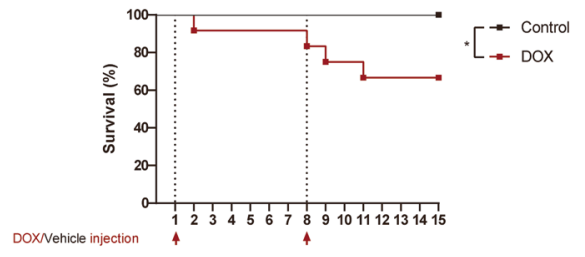

C
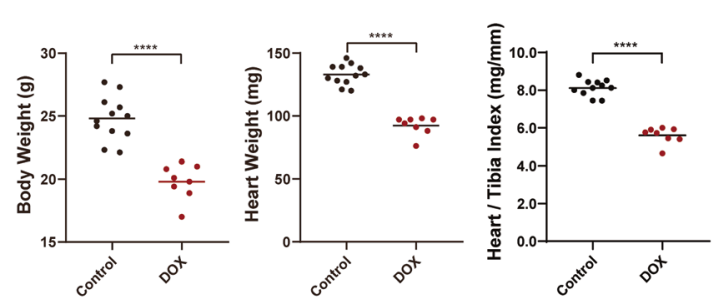

E
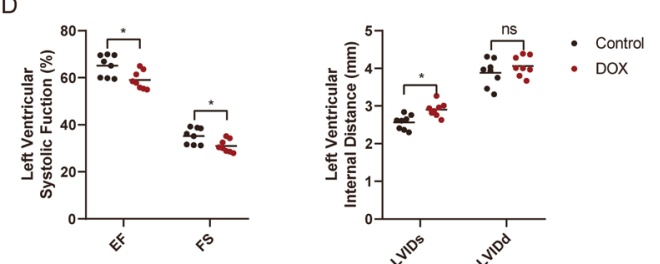
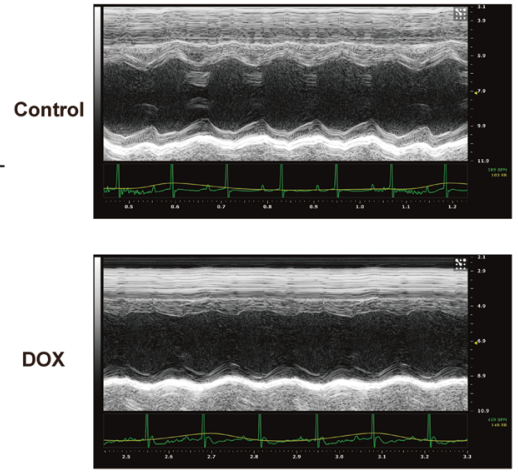

Fig. 1 DOX causes significant cardiac dysfunction and death in mice. a The protocol of DOX-induced sub-chronic cardiotoxicity model establishment. Mice were treated with saline (vehicle control, $N=12)$ or DOX ( $N=12)$ on Day 1 (15 mg/kg DOX, i.p) and Day 8 (10 mg/kg, i.p). b Kaplan-Meier survival curves of control and DOX-treated mice during treatment. c The heart/body weight ratio was measured in control mice and DOX-treated mice. $\mathbf{d}$ Echocardiographic analyses of cardiac function in control mice and DOX-treated mice. EF ejection fraction, FS fractional shortening, LVIDs systolic left ventricular internal diameter, LVIDd diastolic left ventricular internal diameter. e Representative echocardiograms from control and DOX-treated mice on Day 15. Significance in $\mathbf{b}$ was calculated using the log-rank (Mantel-Cox) test. Significance in $\mathbf{c}$, $\mathbf{d}$ was calculated using the unpaired Student's $t$ test. $P$ value $<0.05$ was considered to be significant, and labeled as ${ }^{*} P<0.05$; ${ }^{* * *} P<0.001$; ns not significant. 


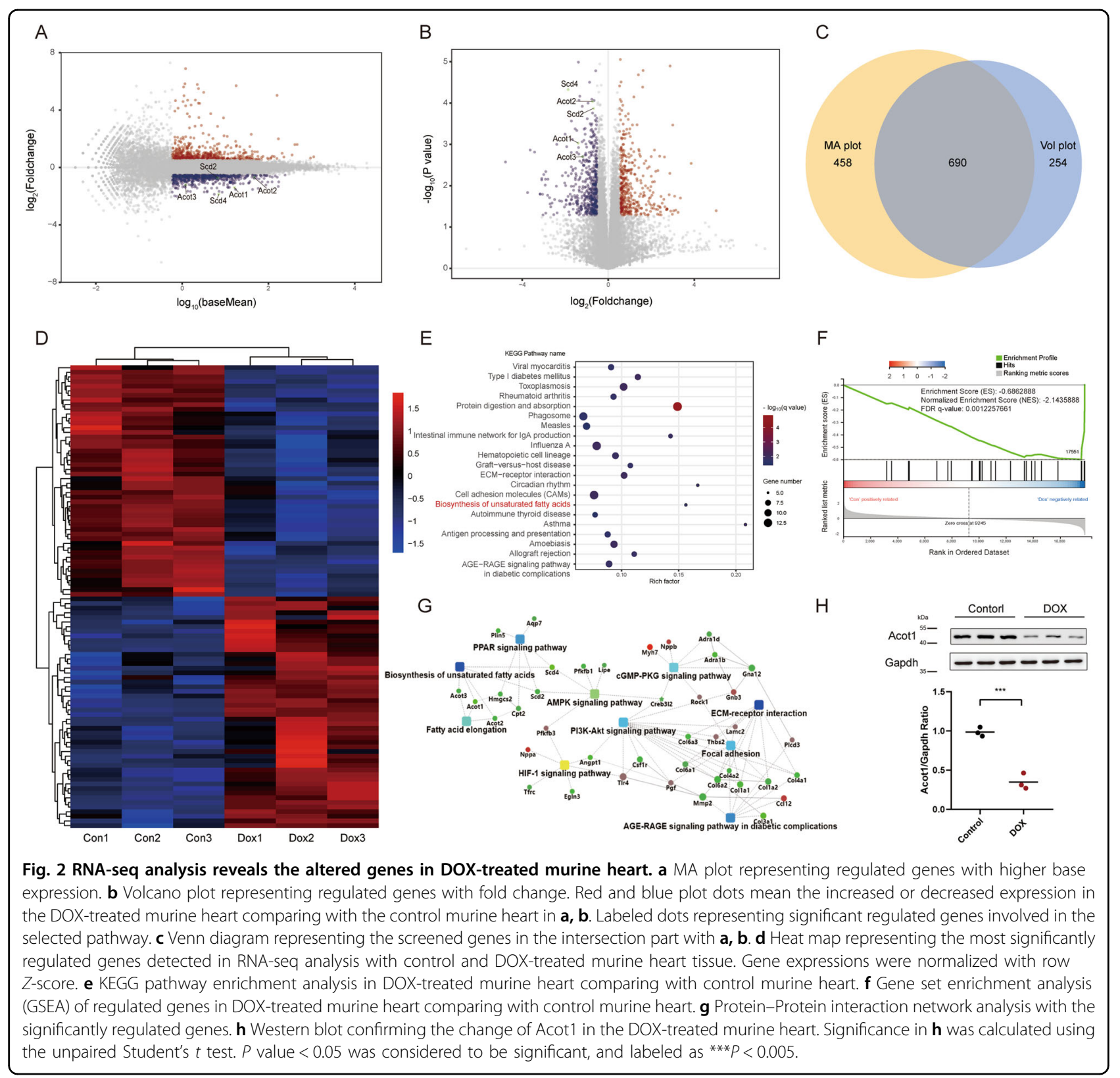

genes (Supplementary Fig. 1). Moreover, the protein level of Acot1 was then confirmed by western blot (Fig. 2h).

Dysregulation of iron metabolism has long been considered to be the primary mechanism that causes cardiomyocytes death in DOX-induced cardiac injury ${ }^{28}$. Taken iron metabolism and biosynthesis of polyunsaturated fatty acid together, a novel pattern of cell death, ferroptosis, come into our mind. Though identified as a novel form of regulated cell death that results from iron-dependent lipid peroxidation, the molecular mechanism of ferroptosis has not yet been fully elucidated, and few studies have been done in cardiovascular diseases ${ }^{29}$. Our subsequent studies then confirmed the existence of ferroptosis in this pathological process. Surprisingly, the RNA-seq analysis did not show significant enrichment in ferroptosis or iron metabolism-related bio-pathways that we currently have known (Supplementary Table 3).

\section{Ferroptosis may participate in the cardiotoxicity induced by DOX}

To further explore the influence of ferroptosis in DOXinduced cardiotoxicity, Fer-1, a selective small molecular inhibitor of ferroptosis, was used to test the cardiac protective function of ferroptosis inhibition in mice treated with DOX. DOX-induced sub-acute cardiac injury model was established with or without Fer-1 intraperitoneal 


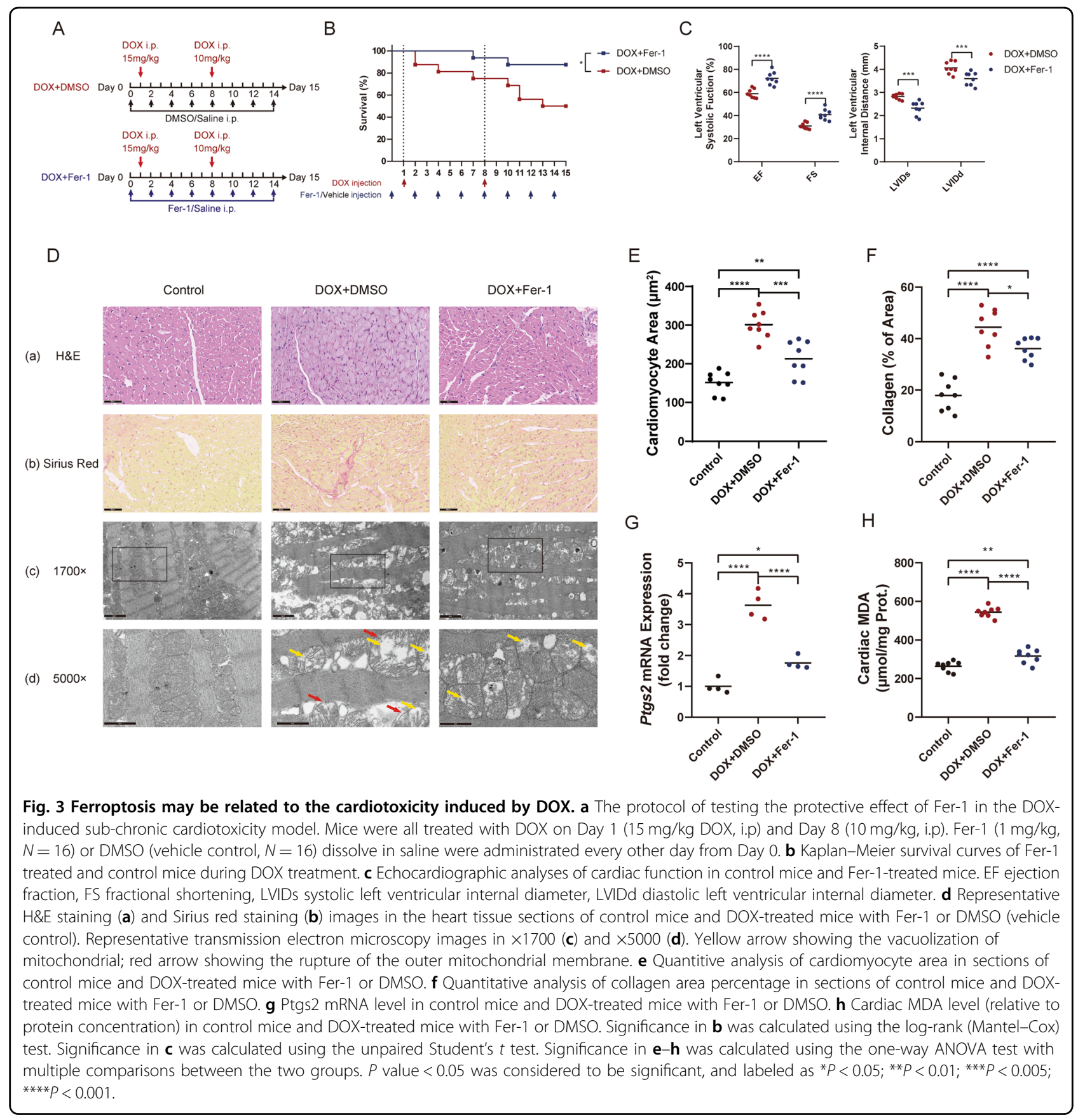

injection every other day (Fig. 3a). Fer-1 treated mice demonstrated a lower death rate and preserved cardiac function (Fig. 3b, c). We then tested the histological changes in DOX-treated murine heart tissue. Comparing with control mice and Fer-1 treated mice, DOX-treated myocardium tissue indicated obvious lipofuscin deposition after 14 days, accompanied by an enlarged crosssectional area due to cardiomyocyte edema (Fig. 3d(a), e and Supplementary Fig. 2a). Also, extensive myocardial fibrosis was observed in the DOX-treated murine heart
(Fig. 3d(b), f). To further validate the existence of ferroptosis, electron transmission microscopy was used to detect the ultrastructure changes of cardiomyocytes. DOX-induced significant myolysis, myofilament loss, and disarrangement in cardiomyocytes, while Fer-1 treatment alleviated these pathological changes (Fig. 3d(c), (d)). Besides, serum cardiac troponin I (cTnI) was used to evaluate the cardiac injury level (Supplementary Fig. 2B).

Meanwhile, Ptgs 2 mRNA expression and cardiac MDA level, both seen as potential ferroptosis signature markers, 


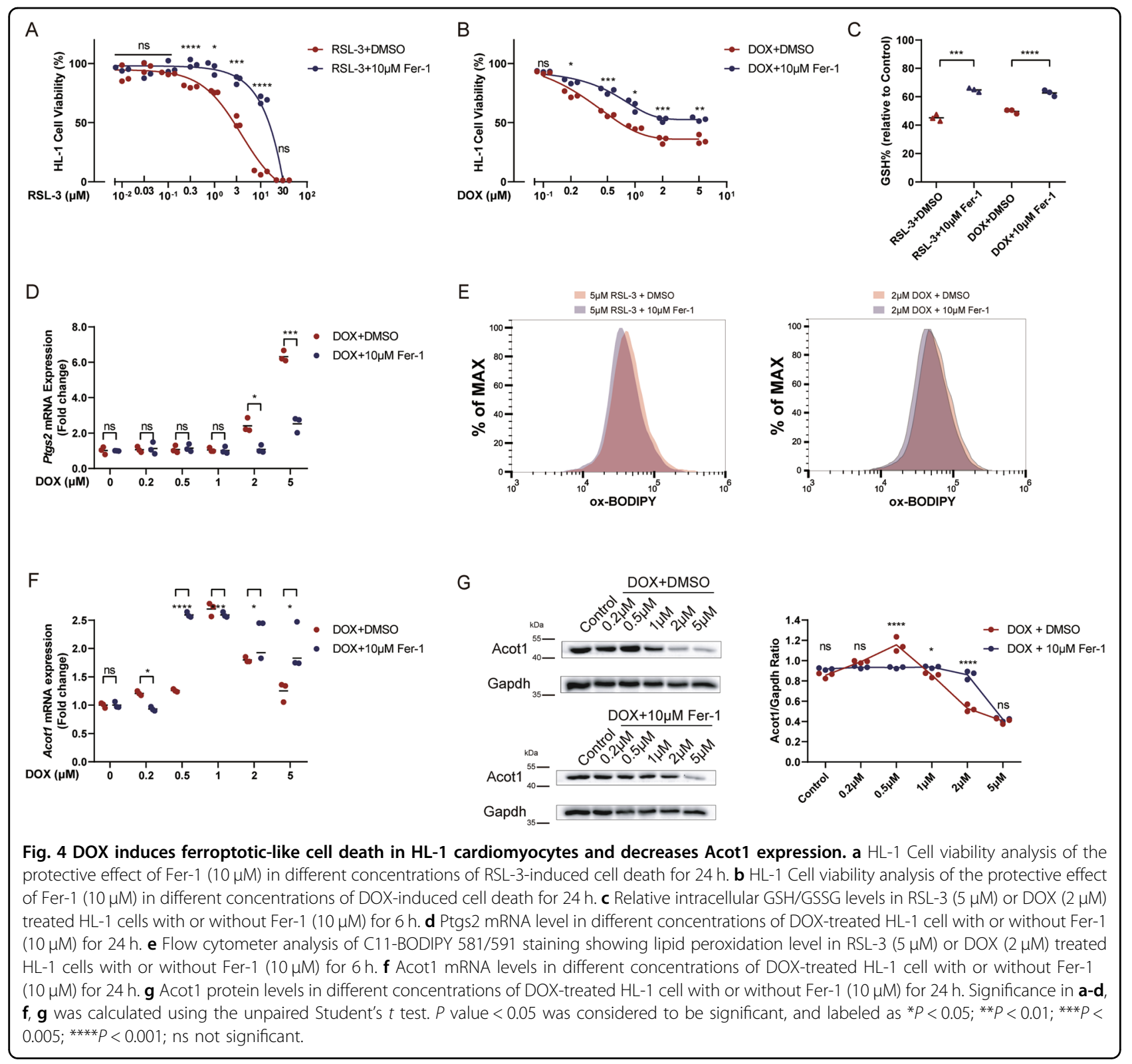

were up-regulated by DOX administration (Fig. 3g, h). We then tested the changes of some ferroptosis-related molecules, including Gpx4, Acsl4, and Fsp1 (Supplementary Fig. 2C). No significant differences were seen among groups.

While apoptosis induced by doxorubicin administration has long been considered as the primary pattern of regulated cell death, which lead to the loss of the cardiomyocytes, we tested the expression level of some apoptosis-related genes. We also explored the changes after the inhibition of ferroptosis. Decreased P53/Gapdh ratio and increased $\mathrm{Bax} / \mathrm{Bcl} 2$ ratio, Cleaved Caspase-3/ Gapdh ratio, and Cleaved Parp1/Gapdh ratio was seen in DOX-treated mice. Fer-1 co-treatment reversed these changes partially. The Caspase-8/Gapdh ratio does not show a significant difference among groups (Supplementary Fig. 3).

These results show that the inhibition of ferroptosis during DOX treatment preserves cardiac function, providing additional evidence that the ferroptosis is involved in this pathological process. Interestingly, inhibition of ferroptosis may also protect cardiomyocytes from apoptosis.

\section{DOX causes ferroptotic-like cell death in HL-1 cardiomyocytes and a reduction of Acot1 expression}

After validating the induction of ferroptosis in DOXtreated mice, we next investigated how Acot1 influenced 


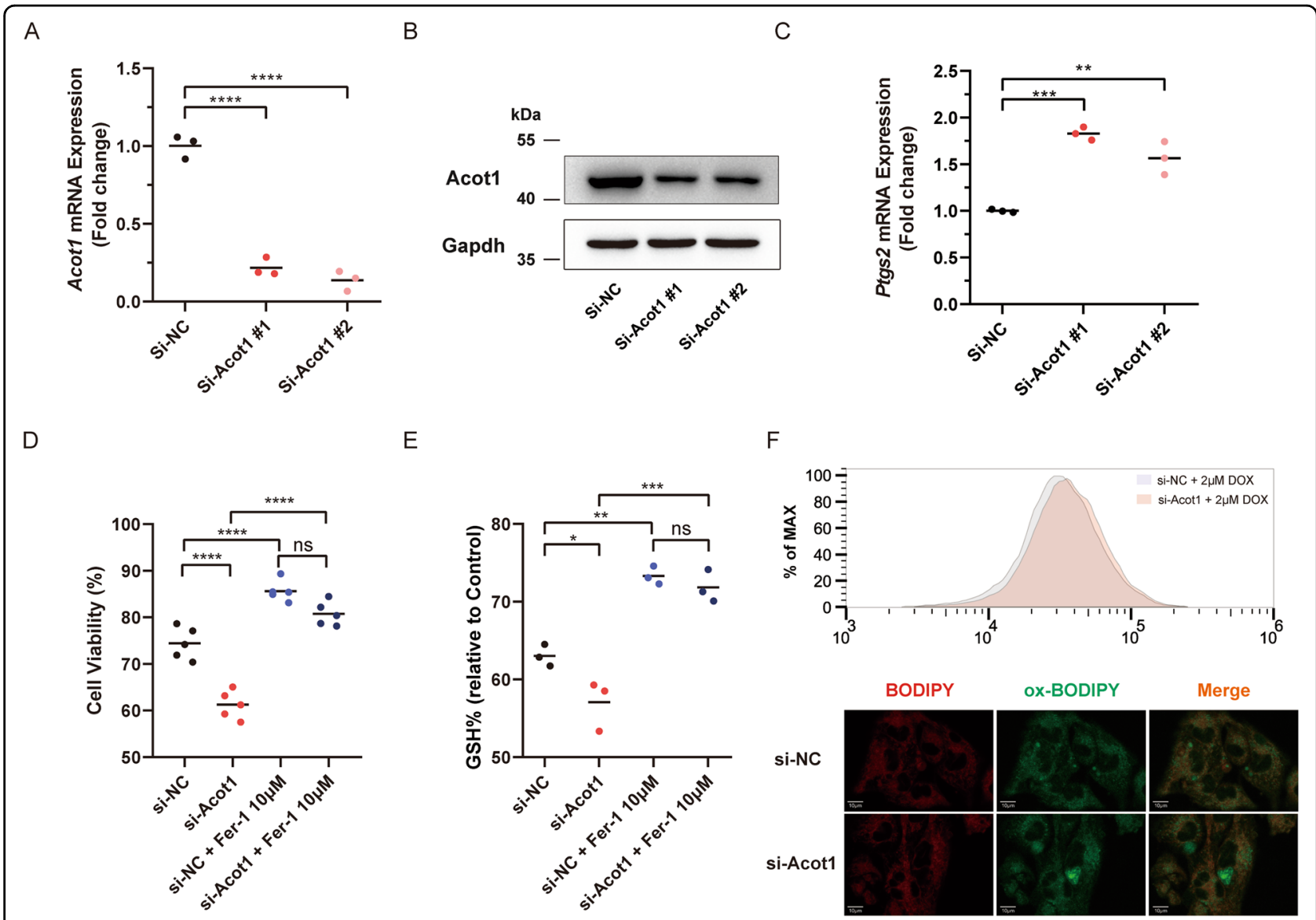

Fig. 5 Acot1 knock-down sensitized cardiomyocytes to DOX toxicity caused by ferroptosis. a Realtime-qPCR confirming the efficiency of siAcot1 \#1, \#2 comparing with scrambling si-NC. b Western blot confirming the efficiency of si-Acot 1 \#1, \#2 comparing with scrambling si-NC. $\mathbf{c}$ Ptgs 2 mRNA level in DOX $(2 \mu \mathrm{M}, 24 \mathrm{~h})$ treated $\mathrm{HL}-1$ cells transfected with si-Acot1 \#1, \#2, and si-NC. d Cell viability analysis showing the effect of Acot1 knock-down in DOX $(2 \mu \mathrm{M}, 24 \mathrm{~h})$ induced cell death and the protective effect of Fer-1 (10 $\mu \mathrm{M})$ co-treatment. e The intracellular GSH/GSSG levels showing the effect of DOX $(2 \mu \mathrm{M}, 6 \mathrm{~h})$ treated HL-1 cells with or without Acot1 knock-down and the protective effect of Fer-1 (10 $\mu \mathrm{M})$ co-treatment. f Flow cytometer analysis of C11-BODIPY 581/591 staining showing lipid peroxidation level in DOX $(2 \mu \mathrm{M}, 6 \mathrm{~h})$ treated HL-1 cells with or without Acot1 knock-down and the representative fluorescent images showing lipid peroxidation level. Significance in $\mathbf{a}, \mathbf{c}$ was calculated using the unpaired Student's $t$ test. Significance in $\mathbf{d}$, e was calculated using the one-way ANOVA test with multiple comparisons between two groups. $P$ value $<0.05$ was considered to be significant, and labeled as ${ }^{*} P<0.05$; ${ }^{* *} P<0.01$; ${ }^{* *} P<0.005 ;{ }^{* * *} P<0.001$; ns not significant.

the process in cardiomyocytes. RSL-3, a class II small molecular inducer of ferroptosis, was used to establish the ferroptosis model in HL-1 cell lines ${ }^{30,31}$. We evaluated the cytotoxic ability of RSL-3 in a concentration gradient with or without Fer-1. Also, the DOX-induced cardiomyocyte death model was established using a similar method. A significant increase of cell death by DOX and RSL-3 administration was detected in a dose-dependent way, while Fer-1 co-treatment inhibited the tendency (Fig. 4a, b). We then measured glutathione levels (GSH and GSSG) in HL-1 cells treated with RSL-3 and DOX. The ratio of GSH/total glutathione showed that both RSL-3 and DOX treatment decreased the antioxidant ability of cardiomyocytes (Fig. 4c). DOX treatment could induce ferroptosis as verified by the elevation of Ptgs 2 mRNA level in $2 \mu \mathrm{M}$, and $5 \mu \mathrm{M}$ DOX-treated HL- 1 cells, while Fer- 1 co- treatment inhibited the elevation of Ptgs2 (Fig. 4d). Lipid peroxidation measured with C11-BODIPY 581/591 probe was recognized as the gold standard for the presence of ferroptosis. As demonstrated, both RSL-3 and DOX treatment caused the significant generation of lipid ROS, which could be inhibited by Fer-1 co-treatment (Fig. 4e). Therefore, Fer-1 treatment may inhibit ferroptosis via anti-lipid peroxidation ability and subsequently decrease DOX caused cell death. Moreover, DOX induces cytotoxicity in HL-1 cells in a pattern similar to RSL-3 induced ferroptosis cell death. We finally tested the influence of DOX treatment on the Acot1 expression. As shown, Acot1 expression was decreased at a higher concentration of DOX, while Fer-1 co-treatment partially suppressed this trend (Fig. 4f, g). Thus, we propose that Acot1 may be a potential protective factor of ferroptosis. 


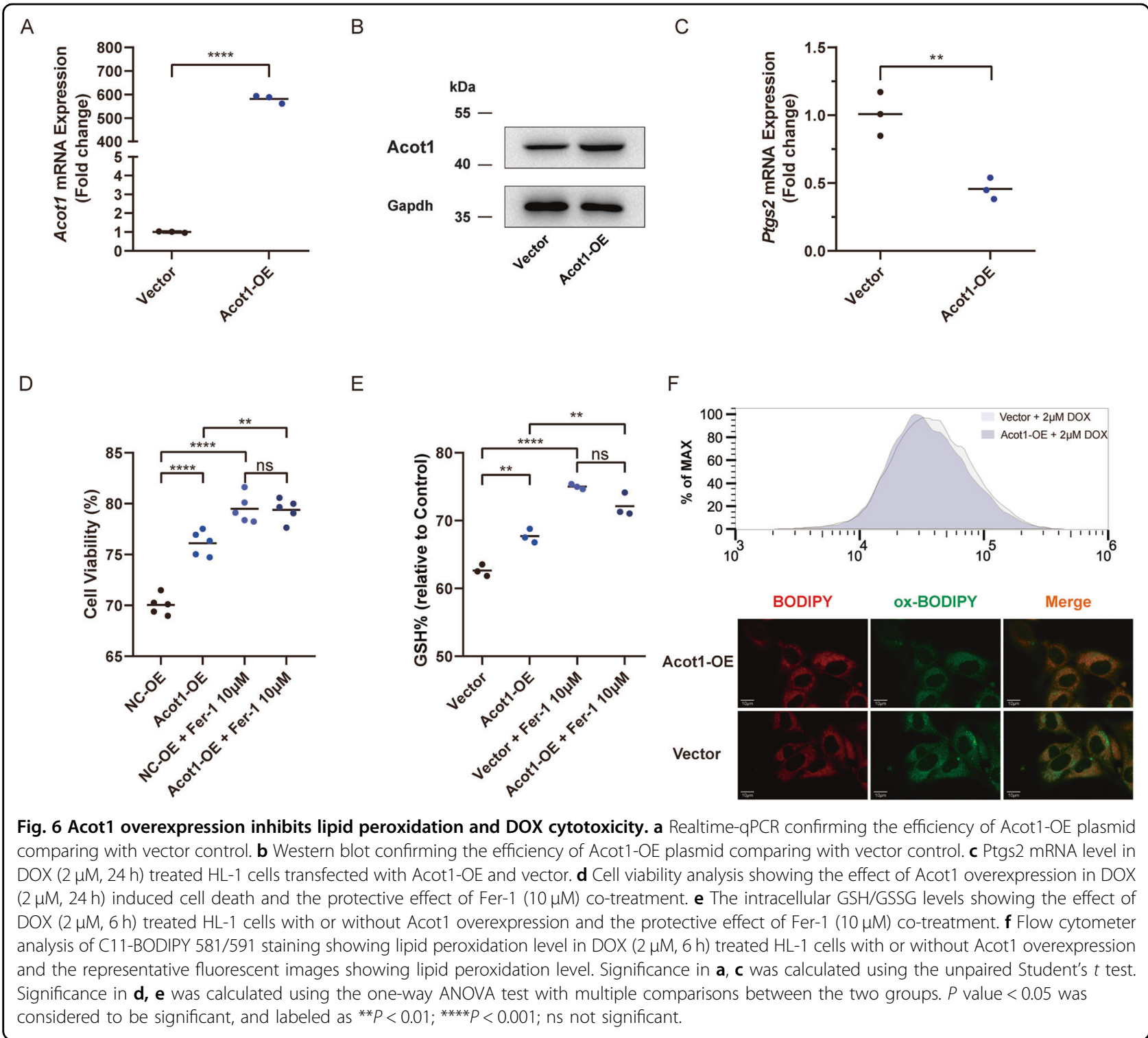

\section{Acot1 knock-down sensitizes cardiomyocytes to DOX toxicity caused by ferroptosis}

As reported before, Acot1 serves as a primary player in the catalytic hydrolysis of acyl-CoA to free fatty acids and $\mathrm{CoA}-\mathrm{SH}^{11}$. Acsl4, a functionally opposite enzyme of Acot1 in lipid metabolism that catalyzes the synthesis of arachidonyl-CoA, was reported to play a role in the induction of ferroptosis ${ }^{32}$. Considering the reduced expression of Acot1 by DOX shown in our study, we think that Acot1 may have the potential ability to inhibit cell ferroptotic death. To verify it, we then examined whether knock-down Acot1 influenced the sensitivity of HL-1 cells to DOX toxicity. The interference efficiency of each siRNA was determined by real-time PCR and western blot independently, and $\mathrm{Si}$-Acot1 \#1 was selected for the further experiment (Fig. 5a, b). Real-time PCR show an elevated Ptgs 2 mRNA level in Acot1 knock-down cardiomyocytes treated with DOX (Fig. 5c). Cell viability assay showed that reduced expression of Acot1 increased cell death induced by DOX treatment, which still could be rescued by Fer-1 co-treatment (Fig. 5d). Decreased GSH/ total glutathione ratio was also observed in Acot1 knockdown cells comparing with scramble control (Fig. 5e). Acot1 knock-down caused more lipid peroxidation as tested by C11-BODIPY staining after DOX treatment (Fig. 5f). These data demonstrated enhanced sensitivity to DOX toxicity after Acot1 knock-down in cardiomyocytes.

\section{Acot1 overexpression inhibits lipid peroxidation and DOX cytotoxicity}

Next, we wonder whether the augmentation of Acot1 expression may reduce the cytotoxicity by DOX. Acot1 
overexpression (Acot1-OE) plasmid and vehicle vector were individually transfected into cardiomyocytes. The elevated expression of Acot1 mRNA and protein were verified by comparing Acot1-OE plasmid transfected cell with vector control (Fig. 6a, b). Declined Ptgs2 mRNA level was observed in Acot1 overexpression cardiomyocytes treated with DOX as compared with control (Fig. 6c). Cell death induced by DOX was inhibited by Acot1 overexpression, and Fer-1 treatment still could rescue the cell death induced by DOX (Fig. 6d). Comparing with vector control, Acot1 overexpression elevated GSH/total glutathione ratio, indicating decreased oxidative stress and reserved antioxidant capacity (Fig. 6e). Consistent with the rescue of cell death, Acot1 overexpression inhibited lipid peroxidation in cardiomyocytes, as verified by C11-BODIPY 581/591 staining (Fig. 6f). Altogether, these data show that Acot1 plays an important protective role in the process of DOX caused cell death.

\section{aMHC-Acot1 transgenic mice show altered free fatty acid composition}

Considering the biochemical function of Acot1 and Acsl4, we proposed that the protective effect of Acot1 was due to its ability to shape lipid composition in cardiomyocytes. As reported before, certain kinds of unsaturated fatty acid sensitized cells to ferroptosis ${ }^{33}$. Therefore, Gas chromatography-mass spectrometry (GC-MS) was used to detect the free fatty acid composition in $\alpha M H C$ Acot1 transgenic mice and their littermates. Generally, murine heart tissue contains abundant free fatty acids, due to its energy metabolic preference (Fig. 7a). Acot1 transgenic murine heart tissues show significantly elevated concentrations of C22:6N3 (Docosahexaenoic Acid, DHA) and C18:0 (Stearic acid), but none significance in C18:2N6 (Linoleic acid), C16:0 (palmitic acid) and C20:4N6 (arachidonic acid, AA) concentrations (Fig. 7b). We then tested the free fatty acids induced sensitization of DIC in HL-1. Data showed a decreased cell viability with a gradient of FFA concentration, and DHA treatment significantly sensitized HL-1 cells to DIC (Fig. 7c). While Fer-1 co-treatment showed a protective effect, Acot1 overexpression also desensitized cardiomyocytes to DHA and AA enhanced DIC (Fig. 7d). Thus, the protective role of Acot1 in DIC was possibly due to the enzymatic function that regulates the free fatty acid composition and distribution in cardiomyocytes, particularly DHA, and then induces desensitization of cardiomyocytes to ferroptosis.

\section{Discussion}

Regulated cell death in terminally differentiated cardiomyocytes plays an important role and has been one of the major interests of investigation in many cardiac pathologies, including DIC $^{32,34}$. Results that inhibition of any one of previously described mechanisms failed to inhibit DIC completely indicate the presence of other unrevealed mechanisms in DIC, which may be related to the dysregulation of iron homeostasis and oxidative stress $^{3,35,36}$. Recently, a research group creatively demonstrated the presence of ferroptosis in the DIC process $^{10}$.

Ferroptosis, as a new form of cell death discovered in 2012, results from iron-dependent lipid peroxide accumulation ${ }^{7}$. While still in its early era, its complete molecular mechanism and its role in disease have remained to be elucidated. Till now, the best-known mechanisms that have been related to ferroptosis were: (1) Excess lipid peroxidation induced by Fenton's reaction (iron-catalyzed oxidative reaction) or lipoxygenase family ${ }^{33,37}$; (2) Lipid hydroperoxides accumulation caused by dysfunction of System $\mathrm{x}_{\mathrm{c}}{ }^{-} / \mathrm{Gpx} 4$ system $^{30,38}$.

Researches in ferroptosis mainly focused on the pathological process of the tumor, yet few of them promoting the important role of ferroptosis in the cardiac disease. In a study of myocardial infarction, the transcription factor $\mathrm{BTB}$ domain and $\mathrm{CNC}$ homolog 1 $(\mathrm{BACH} 1)$ regulate and further influence a set of genes that attenuate ferroptosis, including glutamate-cysteine ligase modifier subunit (Gclm), ferritin heavy chain 1 (Fth1), ferritin light chain 1 (Ftl1), solute carrier family 7 member 11 (Slc7a11), and solute carrier family 40 member 1 $\left(\right.$ Slc40a1) ${ }^{39}$. In another study of DIC, Nrf2/Hmox1 axis has been considered as a pro-ferroptosis factor, due to their ability to rapid conversion heme to free iron and thereby induce iron accumulation in cardiac tissue ${ }^{10}$. These findings encourage us to further explore the importance and relevant mechanisms of ferroptosis in cardiac diseases, such as DIC.

We firstly tested and proved the presence and value of ferroptosis in DIC. As shown in the study, ferroptotic-like morphological changes, characterized by the reduction or vanishing of mitochondria crista and outer mitochondrial membrane rupture ${ }^{40}$, were observed in the DOX-treated murine heart with transmission electron microscopy. Biochemical features of ferroptosis including increased lipid peroxidation was also confirmed by increased MDA concentration and by elevated Ptgs 2 mRNA level ${ }^{41}$. Moreover, DOX-treated mice presented reduced cardiac systolic function and increased death rate, while Fer-1 cotreatment reduced the cardiotoxicity and mortality by DOX. Fer-1 is a small molecular inhibitor of ferroptosis, and its anti-ferroptotic activity is related to the scavenging of ferrous iron-initiated lipid hydroperoxides ${ }^{42}$. The protective effect of Fer-1 to DIC also suggests that ferroptosis may be one of the fundamental mechanisms in the process.

We then wondered what would be the core player of ferroptosis. Some well-known modulators of ferroptosis 

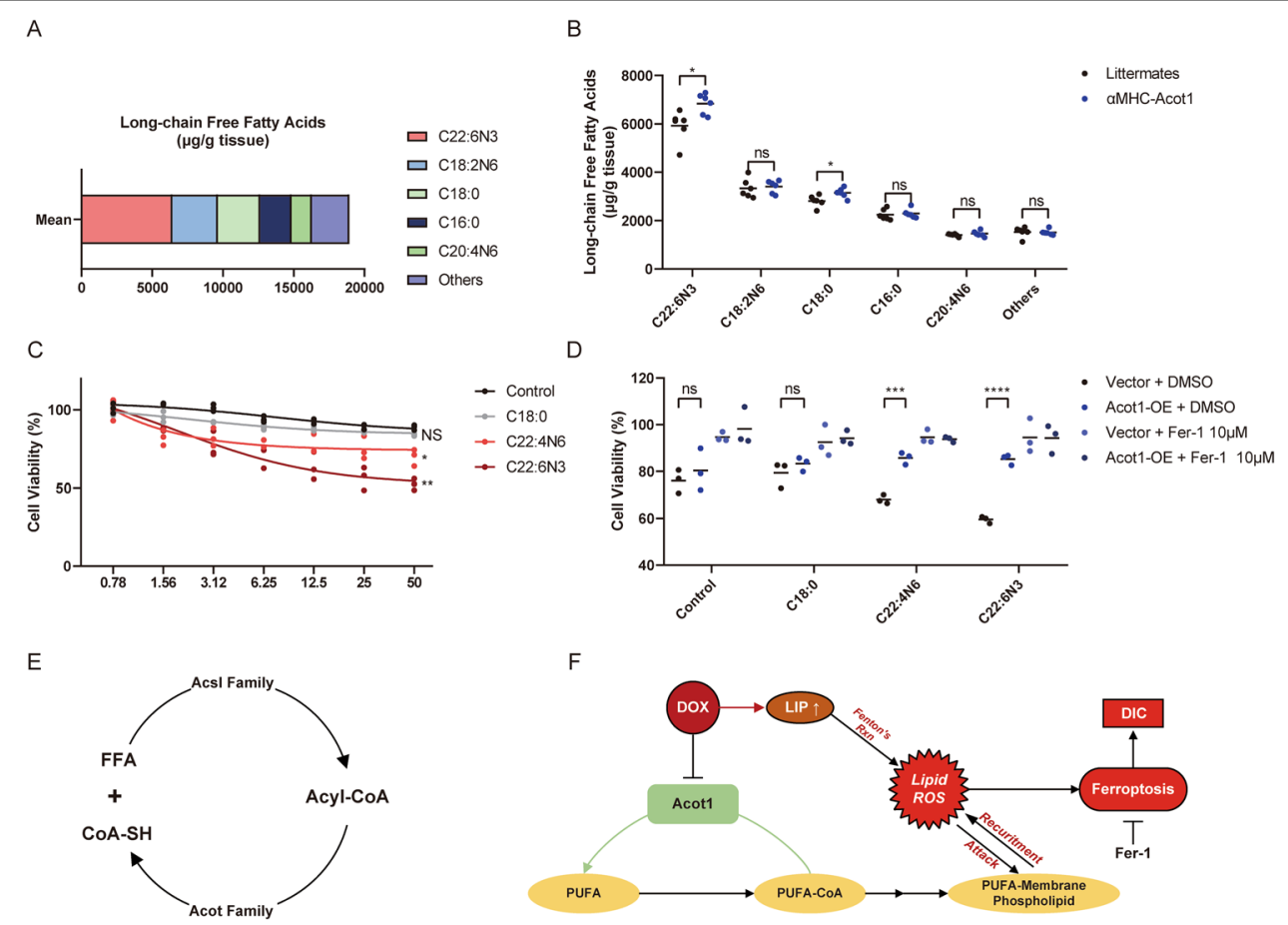

Fig. 7 aMHC-Acot1 transgenic mice show reshaped free fatty acid composition. a The mean concentrations of free fatty acids detected by GCMS in aMHC-Acot1 transgenic mice and their littermates. b Top 5 FFAs concentrations in murine heart tissue. c Cell viability analysis showing different concentrations of FFAs enhanced DIC in HL-1 cells. $\mathbf{d}$ Cell viability analysis showing the protective effect of Acot1 overexpression and Fer-1 co-treatment to FFAs enhanced DIC. e Diagrammatic method of showing the opposite enzymatic function of Acsl family and Acot family in the cell. f The potential mechanism of Acot1 inhibits the induction of ferroptosis in DOX-induced cardiotoxicity. LIP liable iron pool, DIC doxorubicin-induced cardiotoxicity, Fer-1 Ferrostatin-1. Significance in $\mathbf{b}$ was calculated using the unpaired Student's $t$ test. Significance in $\mathbf{c}$, $\mathbf{d}$ was calculated using the one-way ANOVA test with multiple comparisons between the two groups. $P$ value $<0.05$ was considered to be significant, and labeled as $* P<0.05$; ${ }^{* *} P<0.01 ;{ }^{* *} P<0.005 ;{ }^{* * *} P<0.001$; ns not significant.

were tested. Gpx4 and FSP1 (previously known as apoptosis-inducing factor mitochondrial 2 (Aifm2)) were both identified as ferroptosis suppression factors, while Acsl4 was found to induce the sensitivity to ferropto$\mathrm{sis}^{30,43-45}$. However, no significant differences in these genes were seen among groups. A previous study has pointed out that acyl-CoA synthetase long-chain family member-4 (Acsl4) was required for ferroptosis activation. At the same time, Acsl4 deficiency shows great resistance to ferroptosis and significantly reduces the formation of lipid-ROS ${ }^{46}$. The role of ACSL4 in the ferroptotic cell death process rests upon its ability to ligate coenzyme A to long-chain PUFAs, especially with a preference of arachidonic acid (AA) and adrenic acid (AdA), dictate ferroptosis sensitivity by shaping cellular membrane lipid composition $^{33}$. Thus, we speculated that genes modifying the composition of PUFA in the membrane lipids might manipulate the sensitivity to the cell damage caused by ferroptosis inducers as doxorubicin.

The transcriptome sequencing and bioinformatics analysis in the study proved our speculation. With the pathway enrichment analysis and GSEA after RNA-sequencing in the current study, significant downregulation was observed in the pathway of biosynthesis of unsaturated fatty acid in the DOXtreated murine heart. In the sub-acute phase after DOX administration, the protective mechanisms in cardiomyocytes were thought to be inhibited. The PPI-analysis including Gene Ontology (GO) annotation did not screen out a significant hub-gene but provided clues for the possible bio-pathways involved in the pathological process with the most regulated genes. As mentioned in other studies of DIC, the AMPK signaling pathway was also down-regulated in the DOXtreated murine heart and therefore is a potential protective target ${ }^{47}$. Comparing with Scd4, Acot1 has a higher base expression level and mainly localized in the cytosol of cells. Considering the likely mechanism in ferroptosis and an essential role of Acsl4 in it, we then chose Acot1, which catalyze the opposite molecular process of Acsl4, as our major target (Fig. 7e). Moreover, our previous studies have figured out that Acot1 may cast a cardioprotective effect in the process of LPS-induced cardiomyopathy and diabetic cardiomyopathy by decreasing the oxidative stress in cardiomyocytes.

Results from the study by overexpression of Acot1 or knock-down Acot1 verified the beneficial role of Acot1 in DIC. Our previous studies have reported that Acot1 
overexpression prevents cardiac dysfunction in both LPSinduced cardiomyopathy and diabetic cardiomyopathy by reducing oxidative stress ${ }^{14,15}$. Different from them, our current data demonstrated that Acot1 might cast a protective effect in DIC via inhibition of ferroptosis.

Unlike the preferences of Acsl4 in lipid metabolism, an elevated concentration of DHA in the heart tissues of aMHC-Acot1 transgenic mice was observed in the study, yet still as potential substrates for causing ferroptosis. We then confirmed that DHA treatment could sensitize cells to DIC. Surprisingly, we also found that Acot1 overexpression in vitro reduced cell death by DHA. The conflict of exogenous free DHA sensitizes cardiomyocytes to ferroptosis, while Acot1 increases DHA level but prevents DIC may be explained as the augmented PUFA ratio in the bio-membrane structure. The whole process may be regulated by a series of lipid metabolism enzymes, including the synthesis of DHA-CoA and the insertion of DHA-CoA into the bio-membrane ${ }^{9,46}$. In line with the findings in Acot1 transgenic murine heart tissue, Acot1 overexpression in cardiomyocytes may interrupt the regulated process and induce the alteration of lipid composition in cardiomyocytes. Thus, the protective effect of Acot1 on ferroptosis may derive from the altered bio-membrane fatty acids composition of cardiomyocytes in DIC.

Additionally, other lipid metabolism-related genes screened in the current study may also play an important role in the DIC process. Stearoyl-CoA desaturase (Scd) family catalyze the desaturation of saturated acyl-CoA, and Scd1 was nearly reported to be a protective factor in ovarian cancer cells from ferroptotic cell death induced by RSL- ${ }^{48,49}$. The role of Scd family, specifically Scd4 and Scd2, in the induction of DIC needs further investigation.

What is noteworthy is that ferroptosis does perform an important, but not the only player in the pathology process of DIC. Other than the ROS-based mechanism, Top $2 \beta$ in cardiomyocytes may also be a susceptible candidate to $\mathrm{DIC}^{5}$. Ferroptosis inhibition can but only partially rescue the cardiotoxicity of DOX in both in vitro and in vivo experiments. Our data show Fer-1 treatment not only rescue cardiomyocytes from ferroptotic cell death but also reduced the induction of cardiomyocytes apoptosis. To our best knowledge, the process of DIC contains various cell death mechanisms, including but not limited to autophagy, senescence, and pyroptosis. The current work confirmed the presence of ferroptosis in the net-linked mechanisms of DIC, while the cross-talk between these mechanisms still need a series of further investigations.

In conclusion, our study provides evidence suggesting the protective effect of Acot1 in the process of DIC. The underlying mechanism may be related to the reshaping of free fatty acids composition by Acot1 and subsequently desensitization of cardiomyocytes to ferroptosis (Fig. 7f). Further studies will be needed to verify whether Acot 1 is a suitable protective target in DIC.

\section{Acknowledgements}

This work was supported by the National Natural Science Foundation of China (number: 81570367)

\section{Author details}

'Division of Cardiology, Department of Internal Medicine, Tongji Hospital, Tongji Medical College, Huazhong University of Science and Technology, 430030 Wuhan, China. ${ }^{2}$ Hubei Key Laboratory of Genetics and Molecular Mechanisms of Cardiological Disorders, 430030 Wuhan, China. ${ }^{3}$ Department of Biochemistry and Molecular Biology, Tongji Medical College, Huazhong University of Science and Technology, 430030 Wuhan, China

\section{Author contributions}

Y.L. and L.Z. equally contributed to the study design, collected data, and drafted the manuscript. Y.Y. and C.C. participated in performing the study. D.W. gave helpful suggestions in drafting the manuscript. H.W. designed and organized the study.

Conflict of interest

The authors declare that they have no conflict of interest.

\section{Publisher's note}

Springer Nature remains neutral with regard to jurisdictional claims in published maps and institutional affiliations.

Supplementary Information accompanies this paper at (https://doi.org/ 10.1038/s41419-020-02948-2).

Received: 7 February 2020 Revised: 10 August 2020 Accepted: 26 August 2020

Published online: 15 September 2020

\section{References}

1. Arola, O. J. et al. Acute doxorubicin cardiotoxicity involves cardiomyocyte apoptosis. Cancer Res. 60, 1789-1792 (2000).

2. Wang, Y. et al. Chemotherapy drugs induce pyroptosis through caspase-3 cleavage of a gasdermin. Nature 547, 99-103 (2017).

3. Zhang, T. et al. CaMKII is a RIP3 substrate mediating ischemia- and oxidative stress-induced myocardial necroptosis. Nat. Med. 22, 175-182 (2016).

4. Cappetta, D. et al. Doxorubicin targets multiple players: a new view of an old problem. Pharmacol. Res. 127, 4-14 (2018).

5. Zhang, S. et al. Identification of the molecular basis of doxorubicin-induced cardiotoxicity. Nat. Med. 18, 1639-1642 (2012).

6. Chandran, K. et al. Doxorubicin inactivates myocardial cytochrome c oxidase in rats: cardioprotection by Mito-Q. Biophys. J. 96, 1388-1398 (2009).

7. Dixon, S. J. et al. Ferroptosis: an iron-dependent form of nonapoptotic cell death. Cell 149, 1060-1072 (2012).

8. Esterbauer, H. \& Zollner, H. Methods for determination of aldehydic lipid peroxidation products. Free Radic. Biol. Med. 7, 197-203 (1989).

9. Dixon, S. J. et al. Human haploid cell genetics reveals roles for lipid metabolism genes in nonapoptotic cell death. ACS Chem. Biol. 10, 1604-1609 (2015).

10. Fang, $\mathrm{X}$. et al. Ferroptosis as a target for protection against cardiomyopathy. Proc. Natl Acad. Sci. USA 116, 2672-2680 (2019).

11. Dongol, B., Shah, Y., Kim, I., Gonzalez, F. J. \& Hunt, M. C. The acyl-CoA thioesterase I is regulated by PPARalpha and HNF4alpha via a distal response element in the promoter. J. Lipid Res. 48, 1781-1791 (2007).

12. Jezek, P. \& Hlavata, L. Mitochondria in homeostasis of reactive oxygen species in cell, tissues, and organism. Int. J. Biochem. Cell Biol. 37, 2478-2503 (2005).

13. Anderson, E. J., Katunga, L. A. \& Willis, M. S. Mitochondria as a source and target of lipid peroxidation products in healthy and diseased heart. Clin. Exp. Pharmacol. Physiol. 39, 179-193 (2012).

14. Xia, C., Dong, R., Chen, C., Wang, H. \& Wang, D. W. Cardiomyocyte specific expression of Acyl-coA thioesterase 1 attenuates sepsis induced cardiac 
dysfunction and mortality. Biochem. Biophys. Res. Commun. 468, 533-540 (2015).

15. Yang, S. et al. Protective effects of Acyl-coA thioesterase 1 on diabetic heart via PPARalpha/PGC1alpha signaling. PLOS ONE 7, e50376 (2012).

16. $\mathrm{Wu}$, J. et al. Effects of heart rate and anesthetic timing on high-resolution echocardiographic assessment under isoflurane anesthesia in mice. J. Ultrasound Med. 29, 1771-1778 (2010)

17. Langmead, B. \& Salzberg, S. L. Fast gapped-read alignment with Bowtie 2. Nat. Methods 9, 357-359 (2012)

18. Li, B. \& Dewey, C. N. RSEM: accurate transcript quantification from RNASeq data with or without a reference genome. BMC Bioinform. 12, 323 (2011).

19. Lai, L. et al. GSKB: A gene set database for pathway analysis in mouse. bioRxiv, https://doi.org/10.1101/082511 (2016).

20. Subramanian, A. et al. Gene set enrichment analysis: a knowledge-based approach for interpreting genome-wide expression profiles. Proc. Natl Acad. Sci. USA 102, 15545-15550 (2005).

21. R Core Team. R: A Language and Environment for Statistical Computing Nienna, Austria, 2019).

22. Ritchie, M. E. et al. limma powers differential expression analyses for RNAsequencing and microarray studies. Nucleic Acids Res. 43, e47 (2015).

23. Yu, G., Wang, L.-G., Han, Y. \& He, Q.-Y. clusterProfiler: an R package for comparing biological themes among gene clusters. OMICS 16, 284-287 (2012).

24. Kassambara, A. ggpubr: 'ggplot2' Based Publication Ready Plots (2019).

25. Wickham, H. ggplot2: Elegant Graphics for Data Analysis (Springer-Verlag New York, 2016).

26. Kolde, R. pheatmap: Pretty Heatmaps (2019).

27. Schindelin, J. et al. Fiji: an open-source platform for biological-image analysis. Nat. Methods 9, 676-682 (2012).

28. Ichikawa, Y. et al. Cardiotoxicity of doxorubicin is mediated through mitochondrial iron accumulation. J. Clin. Invest. 124, 617-630 (2014).

29. Baba, Y. et al. Protective effects of the mechanistic target of rapamycin against excess iron and ferroptosis in cardiomyocytes. Am. J. Physiol. Heart Circ. Physiol. 314, H659-H668 (2018).

30. Yang, W. S. et al. Regulation of ferroptotic cancer cell death by GPX4. Cell 156, 317-331 (2014).

31. Yang, W. S. \& Stockwell, B. R. Synthetic lethal screening identifies compounds activating iron-dependent, nonapoptotic cell death in oncogenic-RASharboring cancer cells. Chem. Biol. 15, 234-245 (2008).
32. Whelan, R. S., Kaplinskiy, V. \& Kitsis, R. N. Cell death in the pathogenesis of heart disease: mechanisms and significance. Annu. Rev. Physiol. 72, 19-44 (2010).

33. Kagan, V. E. et al. Oxidized arachidonic and adrenic PEs navigate cells to ferroptosis. Nat. Chem. Biol. 13, 81-90 (2017).

34. Mishra, P. K. et al. Guidelines for evaluating myocardial cell death. Am. J. Physiol. Heart Circ. Physiol. 317, H891-H922 (2019).

35. Rouault, T. A. The role of iron regulatory proteins in mammalian iron homeostasis and disease. Nat. Chem. Biol. 2, 406-414 (2006).

36. Canzoneri, J. C. \& Oyelere, A. K. Interaction of anthracyclines with iron responsive element mRNAs. Nucleic Acids Res. 36, 6825-6834 (2008).

37. Yang, W. S. et al. Peroxidation of polyunsaturated fatty acids by lipoxygenases drives ferroptosis. Proc. Natl Acad. Sci. USA 113, E4966-E4975 (2016).

38. Dixon, S. J. et al. Pharmacological inhibition of cystine-glutamate exchange induces endoplasmic reticulum stress and ferroptosis. Elife 3, e02523 (2014).

39. Nishizawa, $\mathrm{H}$. et al. Ferroptosis is controlled by the coordinated transcriptional regulation of glutathione and labile iron metabolism by the transcription factor BACH1. J. Biol. Chem. 295, 69-82 (2020).

40. Xie, Y. et al. Ferroptosis: process and function. Cell Death Differ. 23, 369-379 (2016).

41. Wang, $\mathrm{H}$. et al. Characterization of ferroptosis in murine models of hemochromatosis. Hepatology 66, 449-465 (2017).

42. Miotto, G. et al. Insight into the mechanism of ferroptosis inhibition by ferrostatin-1. Redox Biol. 28, 101328 (2020)

43. Doll, S. et al. ACSL4 dictates ferroptosis sensitivity by shaping cellular lipid composition. Nat. Chem. Biol. 13, 91-98 (2017).

44. Doll, S. et al. FSP1 is a glutathione-independent ferroptosis suppressor. Nature 575, 693-698 (2019).

45. Friedmann Angeli, J. P. et al. Inactivation of the ferroptosis regulator Gpx4 triggers acute renal failure in mice. Nat. Cell Biol. 16, 1180-1191 (2014).

46. Doll, S. et al. ACSL4 dictates ferroptosis sensitivity by shaping cellular lipid composition. Nat. Chem. Biol. 13, 91-98 (2017).

47. Liu, D. et al. AMPK/PGC1a activation by melatonin attenuates acute doxorubicin cardiotoxicity via alleviating mitochondrial oxidative damage and apoptosis. Free Radic. Biol. Med. 129, 59-72 (2018).

48. Tesfay, L. et al. Stearoyl-CoA desaturase 1 protects ovarian cancer cells from ferroptotic cell death. Cancer Res. 79, 5355-5366 (2019).

49. Paton, C. M. \& Ntambi, J. M. Biochemical and physiological function of stearoyl-CoA desaturase. Am. J. Physiol. Endocrinol. Metab. 297. E28-E37 (2009). 